\title{
Congenital Ichthyosis: A Case Treated Successfully With Acitretin
}

\author{
Selvi Gulasi ${ }^{1, *}$ \\ ${ }^{1}$ Department of Pediatrics, Adana Numune Research and Training Hospital, Adana, Turkey \\ "Corresponding author: Selvi Gulasi, Department of Pediatrics, Adana Numune Research and Training Hospital, Adana, Turkey. Tel: +90-5323930542, E-mail: \\ selvigulasi@mynet.com
}

Received 2015 May 21; Revised 2016 January 13; Accepted 2016 April 27.

\begin{abstract}
Introduction: Lamellar ichthyosis (collodion baby) is a cornification disorder classified under the category of autosomal recessive congenital ichthyosis and characterized by hyperkeratosis. Early-stage retinoid treatment has been shown to improve survival in these patients. In this article, a lamellar ichthyosis case is presented of an infant who had the symptoms at birth and was treated successfully with acitretin.

Case Presentation: A term newborn infant presented after delivery. Physical examination showed that the skin on her outer mouth, neck, axillae, and inguinal fold areas had collodion membranes and peelings. On the third day of life, the skin all over her body became dry and seemed similar to parchment paper, with peeling in some areas, as well as ectropion and eclabium development. After her daily bath, liquid Vaseline was applied all over her body, but it did not provide enough benefits. The infant was started on acitretin treatment. On the $14^{\text {th }}$ day of treatment, the skin appeared nearly normal. On the $28^{\text {th }}$ day of life, the infant was discharged.

Conclusions: Early oral retinoid treatment facilitates increased quality of life improves survival rates for ichthyosis patients.
\end{abstract}

Keywords: Ichthyosis, Collodion Baby, Oral Retinoid, Acitretin

\section{Introduction}

Ichthyosis is a group of keratinization disorders that is clinically and etiologically heterogeneous and characterized by dryness, flaking, and peeling of the skin. Due to abnormalities in the structure of the stratum corneum, the increased transepidermal water loss causes excessive dryness. There are congenital and acquired forms of the disease. The congenital forms include lamellar ichthyosis (LI), non-bullous congenital ichthyosiform erythroderma, and Harlequin ichthyosis; the acquired forms include ichthyosis vulgaris and X-linked ichthyosis. LI is a rare congenital anomaly that follows autosomal recessive inheritance (1). Infants are covered with a collodion membrane at birth. After birth, the skin is dry and similar to parchment. The primary treatment consists of moisturizing the skin, reducing fluid loss, and preventing infections. Recently, cases have been reported in which patients have successfully been treated with acitretin, an oral retinoid. Early oral retinoid treatment appears to increase survival rates for these patients (2). In this article, a lamellar ichthyosis case is presented, which was successfully treated with oral acitretin.

\section{Case Presentation}

A term newborn infant presented after delivery due to the detection of peeling on her skin. The patient's prenatal and natal history was insignificant. The parents were second degree relatives. Their other child was healthy, and there was no history of similar diseases in the family. The physical examination showed that the skin on the patient's outer mouth, neck, axillae, and inguinal fold areas had collodion membranes and peelings (Figure 1). Other examination findings were normal.

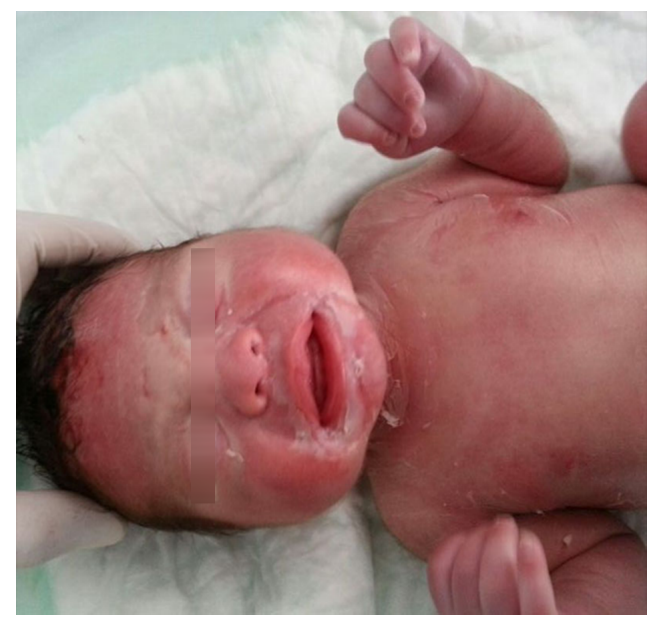

Figure 1. Collodion Membranes and Peeling Were Observed Around the Patient's Mouth, Neck, and Axillary Area Immediately After Birth

On the third day of life, the skin all over the patient's body became dry and seemed similar to parchment paper, with peeling on some areas, as well as ectropion and 


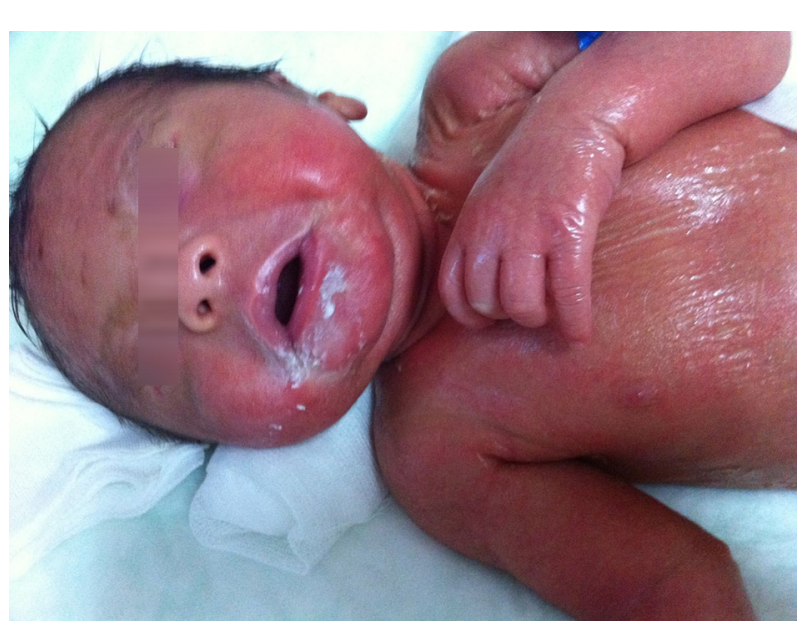

Figure 2. On the 3rd Postnatal Day, Dryness of the Skin, Eclabium on the Lips, and Ectropion on the Eyes Were Observed

eclabium development (Figure 2). After the patient's daily bath, liquid Vaseline was applied all over her body, and ointment containing mupirocin was applied to the open areas of the skin. Drying of the corneas was prevented with artificial tears. Ampicillin $100 \mathrm{mg} / \mathrm{kg} /$ day and gentamicin $4 \mathrm{mg} / \mathrm{kg} /$ day by intravenous route was started because the infant had an increase in infection markers. Fluid treatment was applied according to daily weight and electrolyte level monitoring.

After obtaining the approval of the patient's family, the infant was started on acitretin treatment with a dose of $1 \mathrm{mg} / \mathrm{kg} /$ day by orogastric gavage on the seventh day of life. Biochemical (liver enzymes, renal function tests, electrolytes, serum bilirubin, alkaline phosphatase, serum albumin level, and lipid profile) and hematological (white blood cells, hematocrit and platelets) parameters, which were always evaluated in reference to the normal ranges, were monitored weekly. The abdominal-pelvic and cranial ultrasound, which was performed to check for additional anomalies, was normal. On the $14^{\text {th }}$ day of the treatment, the patient's skin was close to normal (Figure 3). The acitretin treatment of the infant, who had switched completely to oral feeding and recovered from the infection, was changed to every other day. On the $28^{\text {th }}$ day of life, the infant was discharged with recommendations for clinical follow-up. The parents were educated about the disease, and consent was obtained from the family to publish the case and to use the photographs.

\section{Discussion}

LI causes dryness, flaking, and parchment-paper-like peeling on the skin. As in our case, the symptoms are usu- ally present at birth and cover the entire body. These infants are born with a shiny, taught, transparent collodion membrane. The membrane starts to desquamate within one to two weeks. There is usually no erythroderma, and there is no involvement of the mucosa. Due to thickened skin structure and pulling of the soft tissues around the lips and conjunctivae, ectropion and eclabium develop, which are the common diagnostic symptoms in almost all cases. In our case, ectropion and eclabium were both observed. Due to impaired skin integrity, the most significant problems for which these infants are at risk are hyperthermia, increased insensible fluid loss and electrolyte disorders, skin infections, and sepsis (2). Aspiration of the squamous material in the amniotic fluid can cause pneumonia in the first days of life. Sucking and pulmonary ventilation may be hindered.

The diagnosis of LI is made according to clinical symptoms. The clinical course is quite heterogeneous; although $10 \%-20 \%$ of the patients recover with almost no symptoms at all, the rest go through a heavy course of disease (3). The disease may be accompanied by central nervous, immune, endocrine, and/or skeletal system diseases and some malignancies. In our case, no other diseases were detected through physical examination or tests.

LI follows autosomal recessive transmission; its frequency is $1 / 100,000$ (4). There is often a mutation in the gene encoding keratinocyte transglutaminase type 1(TGM 1) (5). TGM 1 encodes the TGAase 1 enzyme, which is one of the three TGAase enzymes found in the epidermis. This enzyme participates in the cornified envelope. In patients with TGM 1 mutation, the cornified envelope is missing, and TGAase 1 activity is reduced or nonexistent. More than

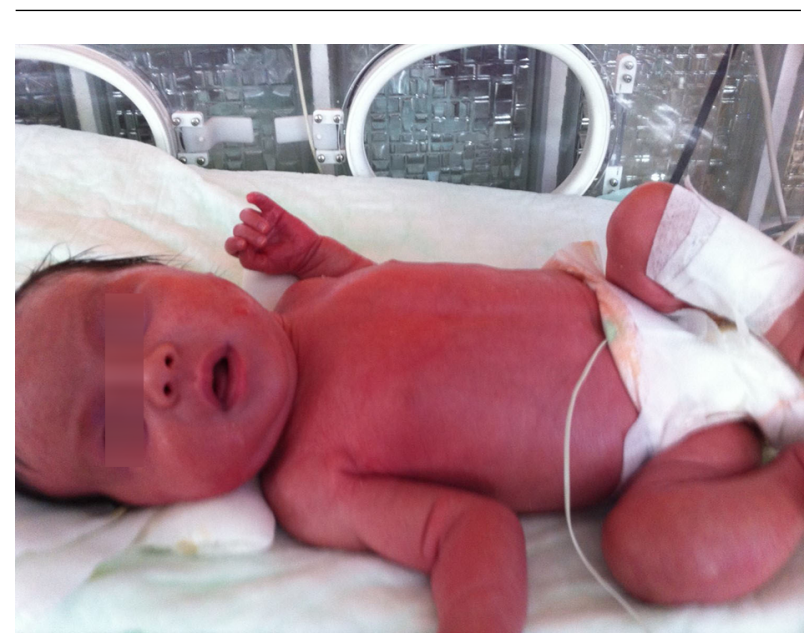

Figure 3. On the $14^{\text {th }}$ day of Acitretin Treatment, the Skin Lesions had Nearly Fully Healed 
110 mutations have been reported (6). ABCA 12 gene mutations have also been reported $(6,7)$. ABCA 12 belongs to a family of $A B C$ transporters, which bind adenosine triphosphate and facilitate the transport of several molecules across the cell membrane. The massive hyperkeratosis that occurs in patients with ABCA 12 mutations could be a compensatory response to a deficient lipid barrier (8).

Neither optical microscopy nor ultrastructural investigations of ichthyosis are specific. It is therefore preferable to delay the skin biopsy until the definitive phenotype has developed. Histopathological changes do not provide a diagnosis. In LI, massive orthokeratotic hyperkeratosis is observed (6).

Prenatal diagnosis through fetal skin biopsy and the microscopic examination of cells in the amniotic fluid can identify families who need genetic counseling for $\operatorname{LI}(7,9)$. Because we could not get permission from the patient's family, we could not perform genetic or histological tests for our patient. Our diagnosis therefore depended on clinical symptoms. Basic treatment of LI includes maintaining proper body temperature with a humidified incubator, maintaining fluid and electrolyte balance and adequate caloric intake, pain control, monitoring of infections, administering baths and moisturizers, eye care with artificial tear drops, and preventing the development of contractures in the distal phalanges (which may cause necrosis and gangrene). Daily bathing is recommended for patients to mechanically eliminate scales and traces of moisturizer (3). Moisturizers and topical keratolytic agents are typically used. Sodium chloride, urea, vitamin E acetate, glycerol, and petroleum can be used as moisturizers (6).

Oral retinoids have keratolytic effects that help eliminate scales and prevent excessive hyperkeratosis (10). Acitretin is a synthetic analog of retinoic acid. Publications regarding its successful use in ichthyosis treatment have increased in recent years. Although its mechanism of action is not precisely known, it is believed that it controls the differentiation and proliferation of the keratinized epithelium. In order to prevent relapse, treatment can take several years for some patients. Long-term use of systemic retinoids has been reported to induce teratogenic and toxic effects in the bone tissue. Bone mineralization may also be affected, and calcification frequently develops in osteophytes and ligaments. Other side effects of oral retinoids include cheilitis, dryness in the mucous membranes, mild hair loss, and itching. Through starting the $1 \mathrm{mg} / \mathrm{kg} /$ day dose of acitretin in our case, whose clinical symptoms were compatible with LI, the skin symptoms were significantly improved during the first week of the treatment, and by the end of the second week, the patient's skin was close to normal. The patient's tolerance for the drug was good. Side effects were not observed.

\subsection{Conclusion}

In this case, the patient showed a good response to oral retinoid treatment for $\mathrm{LI}$, and the patient's quality of life and odds of survival increased. However, larger studies are needed in order to determine acitretin's mechanism of action and its long-term side effects.

\section{Footnote}

Authors' Contribution: Study concept and design, acquisition of data, analysis and interpretation of data, drafting of the manuscript, critical revision of the manuscript for important intellectual content, statistical analysis, no analysis was conducted, administrative, technical, and material support, study supervisor: Selvi Gulasi.

\section{References}

1. DiGiovanna JJ. In: Fitzpatrick's dermatology in general medicine. Freeberg IM, Eisen AZ, Wolf K, editors. McGraw-Hill; 2003.

2. Rajpopat S, Moss C, Mellerio J, Vahlquist A, Ganemo A, Hellstrom-Pigg $\mathrm{M}$, et al. Harlequin ichthyosis: a review of clinical and molecular findings in 45 cases. Arch Dermatol. 2011;147(6):681-6. doi: 10.1001/archdermatol.2011.9. [PubMed: 21339420].

3. Vahlquist A, Ganemo A, Virtanen M. Congenital ichthyosis: an overview of current and emerging therapies. Acta Derm Venereol. 2008;88(1):4-14. doi: 10.2340/00015555-0415. [PubMed: 18176742].

4. Ganemo A, Pigg M, Virtanen M, Kukk T, Raudsepp H, RossmanRingdahl I, et al. Autosomal recessive congenital ichthyosis in Sweden and Estonia: clinical, genetic and ultrastructural findings in eighty-three patients. Acta Derm Venereol. 2003;83(1):24-30. [PubMed: 12636018].

5. Huber M, Rettler I, Bernasconi K, Frenk E, Lavrijsen SP, Ponec M, et al. Mutations of keratinocyte transglutaminase in lamellar ichthyosis. Science. 1995;267(5197):525-8. [PubMed: 7824952].

6. Rodriguez-Pazos L, Ginarte M, Vega A, Toribio J. Autosomal recessive congenital ichthyosis. Actas Dermosifiliogr. 2013;104(4):270-84. doi: 10.1016/j.adengl.2011.11.021. [PubMed: 23562412].

7. Akiyama M, Sugiyama-Nakagiri Y, Sakai K, McMillan JR, Goto M, Arita $\mathrm{K}$, et al. Mutations in lipid transporter ABCA12 in harlequin ichthyosis and functional recovery by corrective gene transfer. J Clin Invest. 2005;115(7):1777-84. doi: 10.1172/JCI24834. [PubMed: 16007253].

8. Zuo Y, Zhuang DZ, Han R, Isaac G, Tobin JJ, McKee M, et al. ABCA12 maintains the epidermal lipid permeability barrier by facilitating formation of ceramide linoleic esters.J Biol Chem. 2008;283(52):36624-35. doi: 10.1074/jbc.M807377200. [PubMed: 18957418].

9. Morelli JG. In: Disorders of keratinization. Kliegman RM, Behrman RE, Jenson HB, Stanton BF, editors. ; 2007.

10. Digiovanna JJ, Mauro T, Milstone LM, Schmuth M, Toro JR. Systemic retinoids in the management of ichthyoses and related skin types. Dermatol Ther. 2013;26(1):26-38. doi: 10.1111/j.1529-8019.2012.01527.x. [PubMed: 23384018]. 